\title{
Endoscopic ultrasound-guided drainage of a pelvic abscess via a J-pouch
}

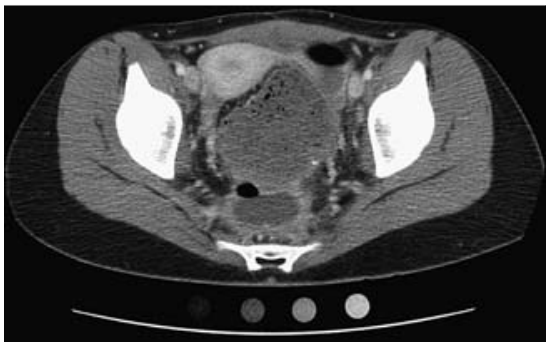

Fig. 1 Computed tomography (CT) of the pelvis, revealing a $5 \times 3-\mathrm{cm}$ pelvic abscess in a patient with J-pouch anatomy.

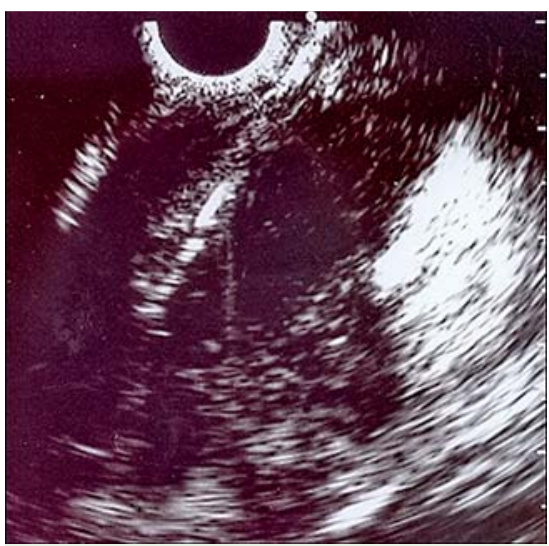

Fig. 2 Endoscopic ultrasound (EUS) image: the abscess cavity was punctured using a 19gauge fine needle aspiration needle via the J-pouch under EUS guidance.

While prior reports have demonstrated the usefulness of endoscopic ultrasound (EUS) for transrectal drainage of pelvic abscesses, its utility for performing drainage via an ileoanal reservoir (J-pouch) has not been reported before.

A 28-year-old patient with a history of total colectomy and a J-pouch for ulcerative colitis presented with persistent fever and rectal pain. Computed tomography (CT) of the pelvis revealed an abscess measuring $5 \times 3 \mathrm{~cm}$ adjacent to the J-pouch ( $\bullet$ Fig. 1 ). EUS-guided drainage of the abscess was requested because of the lack of an adequate window for percutaneous drainage. At EUS, the pelvic abscess was punctured ( $\bullet$ Fig. 2) using a 19-gauge needle (Expect; Boston Scientific, Natick, Massachusetts, USA), and a 0.035 -inch guide wire was then coiled into the abscess ( Fig.3) under fluoroscopic guidance. The transmural tract was sequentially
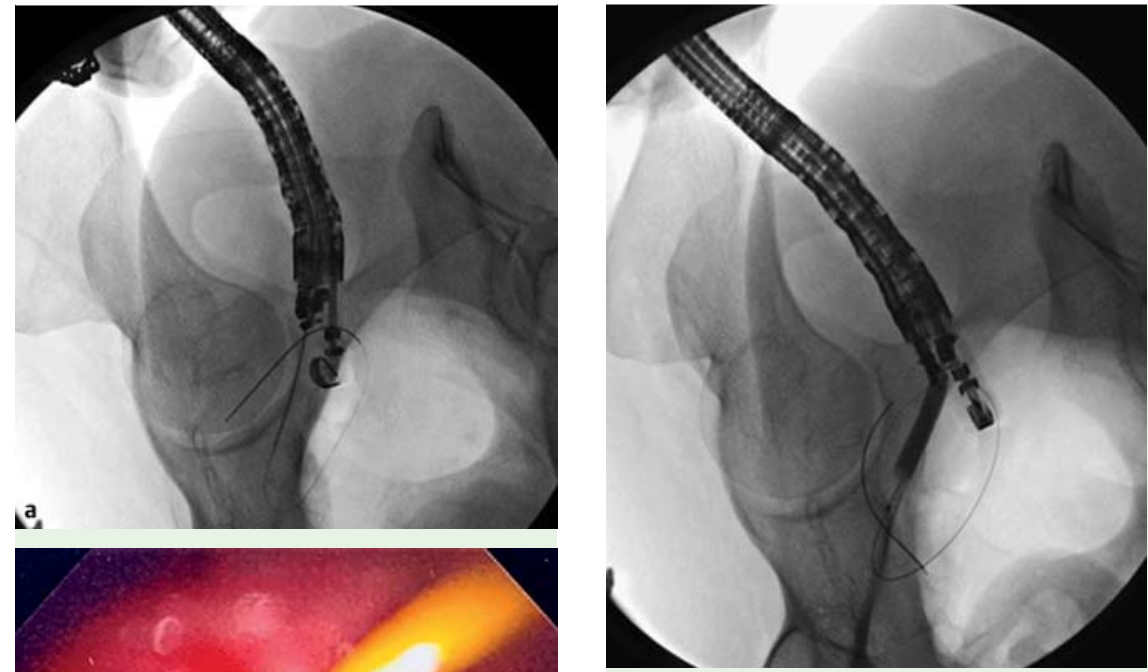

dilated using a 5-Fr endoscopic retrograde cholangiopancreatography cannula and a 6-mm balloon dilator ( Fig. 4). A 7-Fr double pigtail stent was then deployed into the abscess cavity ( $\bullet$ Fig. 5).

Postprocedure, the patient was afebrile and had no rectal pain. Follow-up CT revealed complete resolution of the abscess, and so the transrectal stent was retrieved by sigmoidoscopy.

Fitting a J-pouch, sometimes referred to as ileoanal reservoir, involves colectomy with mucosal proctectomy and the creation of an ileal reservoir which is anastomosed to the anal canal [1]. In a meta-analysis, $9.5 \%$ of patients with a J-pouch developed pelvic abscess from anastomotic dehiscence [2]. Initial management often includes percutaneous drainage; a persist-
Fig. 4 Dilation of the transmural tract using a
6-mm over-the-wire balloon.

Fig. 4 Dilation of the transmural tract using a
6-mm over-the-wire balloon.
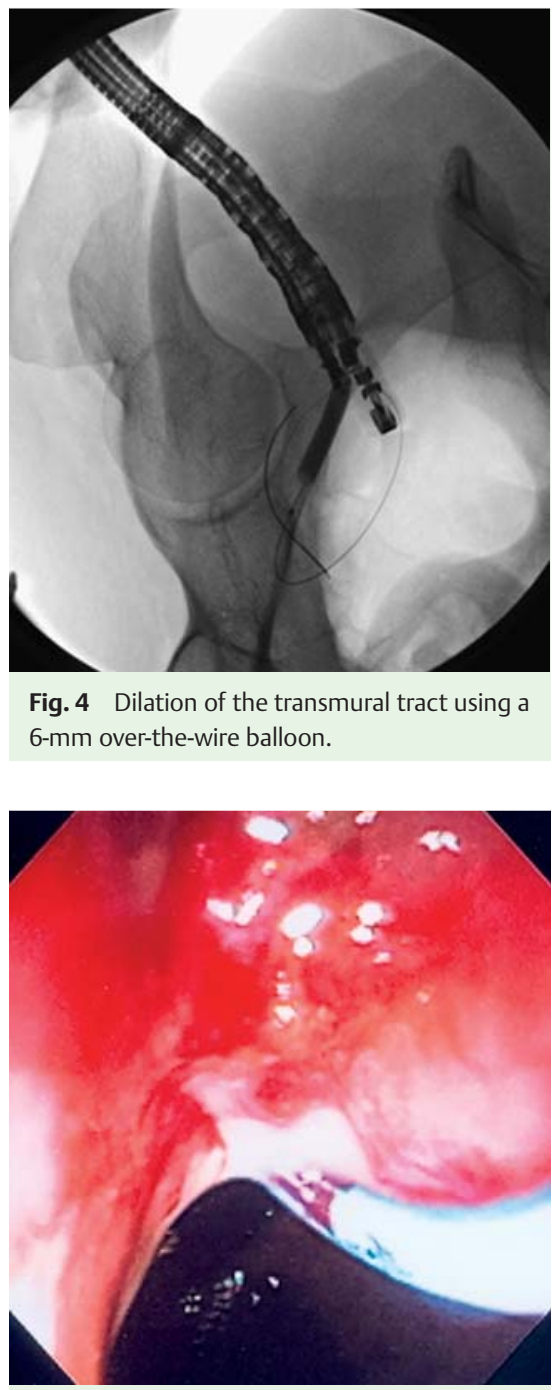

Fig. 5 Placement of a double pigtail stent into the abscess cavity via the J-pouch.

ent abscess may require surgery [3]. In a prior study by myself and a co-author, we have shown that EUS is a minimally invasive alternative for drainage of pelvic abscesses [4]. However, patients with a Jpouch were excluded because of concerns of perforation in a surgically constructed anatomy. Given the inability to treat the pelvic abscess by percutaneous means, we attempted drainage via the J-pouch in this patient, with good clinical outcomes.

\section{Endoscopy_UCTN_Code_TTT_1AS_2AZ}

Competing interests: None 


\section{S. Varadarajulu}

University of Alabama at Birmingham School of Medicine, Birmingham, Alabama, USA

\section{References}

1 Broder JC, Tkacz JN, Anderson SW et al. Ilealpouch-anal anastomosis surgery: imaging and intervention for post-operative complications. Radio Graphics 2010; 30: 221 - 233

2 Hueting WE, Buskens E, van der Tweel I et al. Results and complications after ileal pouch anal anastomosis: a meta-analysis of 43 observational studies comprising 9,317 patients. Dig Surg 2005; 22: 69-79

3 Farouk R, Dozois RR, Pemberton JH et al. Incidence and subsequent impact of pelvic abscess after ileal pouch-anal anastomosis for chronic ulcerative colitis. Dis Colon Rectum 1998; 41: 1239-1243

4 Varadarajulu S, Drelichman ER. Effectiveness of EUS in drainage of pelvic abscesses in 25 consecutive patients (with video). Gastrointest Endosc 2009; 70: 1121 - 1127
Bibliography

DOI $10.1055 / \mathrm{s}-0030-1256946$

Endoscopy 2012; 44: E92 - E93

(c) Georg Thieme Verlag KG Stuttgart · New York . ISSN 0013-726X

\section{Corresponding author}

S. Varadarajulu, MD

Basil I. Hirschowitz Endoscopic

Center of Excellence

University of Alabama at Birmingham

School of Medicine

JT 664, 1530 3rd Avenue South

Birmingham

Alabama 35294

USA

Fax: +1-205-975-6381

svaradarajulu@yahoo.com 